\begin{tabular}{|c|c|c|}
\hline Beitr. Ent. & Berlin & ISSN 0005-805X \\
\hline $\mathbf{5 0}(2000) 2$ & S. $271-281$ & 02.10 .2000 \\
\hline
\end{tabular}

\title{
Anatomische, histologische und histochemische Untersuchungen des männlichen Geschlechtsapparates von Cosmopolites sordidus (GERMAR)
}

\section{(Coleoptera: Curculionidae)}

Mit 7 Figuren

Ali AgHA NAHIF

\section{Zusammenfassung}

Der Bananenrüßler Cosmopolites sordidus (GERMAR) hat sich in weiten Teilen Afrikas zum wichtigsten Schädling der Banane (Musa spp.) entwickelt.

Der männliche Geschlechtsapparat von $C$. sordidus besteht aus Hoden, Vasa deferentia, Vesicula seminales, paarigen akzessorischen Drüsen, Ductus ejaculatorius und Aedeagus. Alle Organe wurden beschrieben und mit Figuren dargestellt. Das Sekret der paarigen akzessorischen Drüsen und der Vasa deferentia besteht aus Proteinen. Die Proteinkonzentration der akzessorischen Drüsen wurde photometrisch bestimmt.

\section{Summary}

The banana weevil Cosmopolites sordidus (GERMAR) has become the most important pest of banana (Musa spp.) in Afrika.

The male reproductive system of $C$. sordidus consists of the testes, the vasa deferentia vesiculae seminales, one pair of accessory glands, the ductus ejaculatorius, and the aedeagus, all of wich are described and illustrated. The secretions of accessory glands and the vasa deferentia mainly consist of proteins. The protein consentration of the accessory glands was measured photometrically.

\section{Key words}

Coleoptera, Curculionidae, banana weevil, Cosmopolites sordidus, internal genitalia

\section{Einleitung}

Der Bananenrüßler Cosmopolites sordidus (GERMAR), einer der bedeutendsten Schädlinge der Banane, ist in weiten Teilen des Kontinents Afrika verbreitet und verursacht Jahr für Jahr große Schäden in den Plantagen (NAHIF u. a., 1994).

In der Literatur finden sich zahlreiche Publikationen über den Bananenrüßler. Die meisten Publikationen befassen sich mit der Bekämpfung dieser Schädlinge (BENDICHO-LOPEZ 1987, Castinerias u. a, 1991, Chavarria-CARvajal \& IRIZARry 1997, Figueroa 1990, Nair 1979, SCHOMANS 1998).

Biologische und morphologische Untersuchungen an C. sordidus liegen von BECCARI (1967), KOPPENHÖFFER (1993), LONGORIA (1968), MAU (1982), MESQUITA \& CALDAS (1986), NAHIF u. a. (1994), NARDON u. a. (1984), VISNAWATH (1981) vor. 
BECCARI (1967) und ZIMMERMANN (1968) gehen in ihren Untersuchungen über die Bananenrüßler im Detail auf den Geschlechtsapparat von C. sordidus ein. Hingegen fehlen Untersuchungen zur Anatomie, Histologie und Histochemie des Geschlechtsapparates von C. sordidus, weshalb solche Untersuchungen zum Gegenstand der vorliegenden Arbeit gemacht wurden und der männliche Geschlechtsapparat von $C$. sordidus beschrieben wird.

\section{Danksagung}

Ich danke den Herren Prof. Dr. RICHARD SIKORA und Dr. HOLGER HiNDORF (Institut für Pflanzenkrankheiten, Bonn) für die Beschaffung des Materials aus Kenya, Herrn Dr. GüNTER MADEL und Herrn Dr. SIEGFRIED STEPHAN (Uni. Bonn) für die kritische Durchsicht des Manuskriptes und Herrn DIETER RoTT (Garten des Botanischen Instituts, Bonn) für die Überlassung des Bananenrhizoms.

\section{Material und Methoden}

Die männlichen und weiblichen adulten Käfer stammen aus Kenya. Sie wurden in Plastikeimern (20 cm Durchmesser und $14 \mathrm{~cm}$ Höhe) mit feuchter Erde auf einem Bananenrhizom im Labor bei Temperaturen zwischen $20-28^{\circ} \mathrm{C}$ und einer Luftfeuchte von $50-60 \%$ gehalten. Aus den um sie herum abgelegten Eiern schlüpften Larven, die sich nach ca. 7 Wochen zu adulten Käfern entwickelten.

Die Präparation der männlichen Tiere erfolgte in einer $0,65 \%$ igen NaCl-Lösung. Die isolierten Geschlechtsapparate wurden in Bouin oder in Formol nach Baker (ROMEIS 1989) bei $+4{ }^{\circ} \mathrm{C}$ fixiert, um Total- und Schnittpräparate anzufertigen. Von den in Paraffin eingebetteten Objekten wurden 5-7 $\mu \mathrm{m}$ dicke Paraffinschnitte hergestellt und zur Färbung der Schnitte wurde neben Eisenhämatoxylin und Azan nach Heidenhain auch die modifizierte Azanfärbung nach Specht (ROMEIS 1989) angewandt. Als optimal erwies sich Azanfärbung nach Heidenhain (ROMEIS 1989).

Zur histochemischen Untersuchungen wurde das Sekret der Vasa deferentia und der akzessorischen Drüsen von $C$. sordidus auf den Gehalt an Kohlenhydraten (Nachweis mit PAS und BESTscher Karmin-Färbung), sauren Mucopolysacchariden (Nachweis mit Alcianblau, Astrablau und Toluidinblau-Färbung), Proteine (Nachweis mit Quecksilber-Bromphenol und FastGreen-Färbung) und schließlich die Lipide (Nachweis mit 1\%iger Osmiumsäure, Sudanschwarz B und Scharlach R-Färbung) geprüft (ROMEIS 1989, RUTHMANN 1966, SPANNHOF 1967). Die gesamte Färbungen wurde an 5-7 $\mu \mathrm{m}$ dicken Paraffinschnitten durchgeführt.

Die fotografische Dokumentation der anatomischen und histologischen Präparate erfolgte am Universalmikroskop (Leitz).

Für die Ermittlung der Proteinkonzentration der akzessorischen Drüsen kam die Methode BCA Protein Assay Reagent Kit der Firma PIERCE (Rockford, Il. USA) zu Anwendung. Diese Methode ist für die Spectrophotometrische Bestimmung der Proteinkonzentration sehr gut geeignet.

Den Drüsenschläuchen $(\mathrm{n}=10)$ wurden $50 \mu \mathrm{l} 0,1 \mathrm{~N}-\mathrm{NaOH}$ zugefügt. Nach der Homogenisierung im Ultraschallwasserbad wurde die Probe abzentrifugiert. In je $10 \mu 1$ des Ansatzes und eine Standdardlösung (Albumin) wurden mit Hilfe des EIA-Datenauswertungsprogramms (ICN-Biomedicals GmbH EIA-Version 3.10) die Proteinkonzentrationen photometrisch (Fr. Titertek Multiskan Plus) bestimmt und die Ergebnisse registriert.

Der Nachweis der Spermien wurde mit dem FluoreszenzfarbstoffDAPI, einem Phenyl-Derivat (4, 6-Diamidoindo-2-Phenylindol) durchgeführt. Das Fluorochrom färbt DNA spezifisch (LIN u. a., 1977). Der Farbstoff wurde in einer Konzentration von $5 \mu \mathrm{g} / \mathrm{ml}$ Tris-Puffer eingesetzt. Dazu wurden die entparaffinierten Schnitte $10 \mathrm{~min}$ mit einer $10^{-3} \mathrm{M}$-DAPI-Lösung über- 
schichtet. Nach Abgießen der überstehenden DAPI-Lösung wurden die Präparate mit KAISERs Glyceringelatine eingedeckelt und das Deckglas mit Nagelöllack fixiert. Die Färbung wirkt sehr schnell und der DAPI-DNA-Komplex fluoresziert weiß. Für die DAPI-Fluoreszierung eignen sich als Filterkombination der Anregungsfilter BP 350-410 und der Sperffilter LP $445 \mathrm{~nm}$.

\section{Ergebnisse}

Der männliche Geschlechtsaparat von C. sordidus füllt den hinteren Teil des Abdomens des adulten Käfers aus. Er ist von zahlreichen Fettkörperlappen bedeckt, sowie von Tracheen und Tracheolen überzogen. Der Geschlechtsapparat weist jeweils paarige Hoden auf. Jedem Hodenpaar sind eine blind endende akzessorische Drüse, ein Vesiculum seminalis und ein Ductus ejaculatorius zugeordnet. Beide Ducti ejaculatorius laterales vereinigen sich zu einem unpaaren Ductus ejaculatorius, der in den Aedeagus einmündet (Fig. 1A). Im distalen Bereich ist der gewundene, einsträngige Ductus ejaculatorius von kräftiger Muskulatur umgeben.

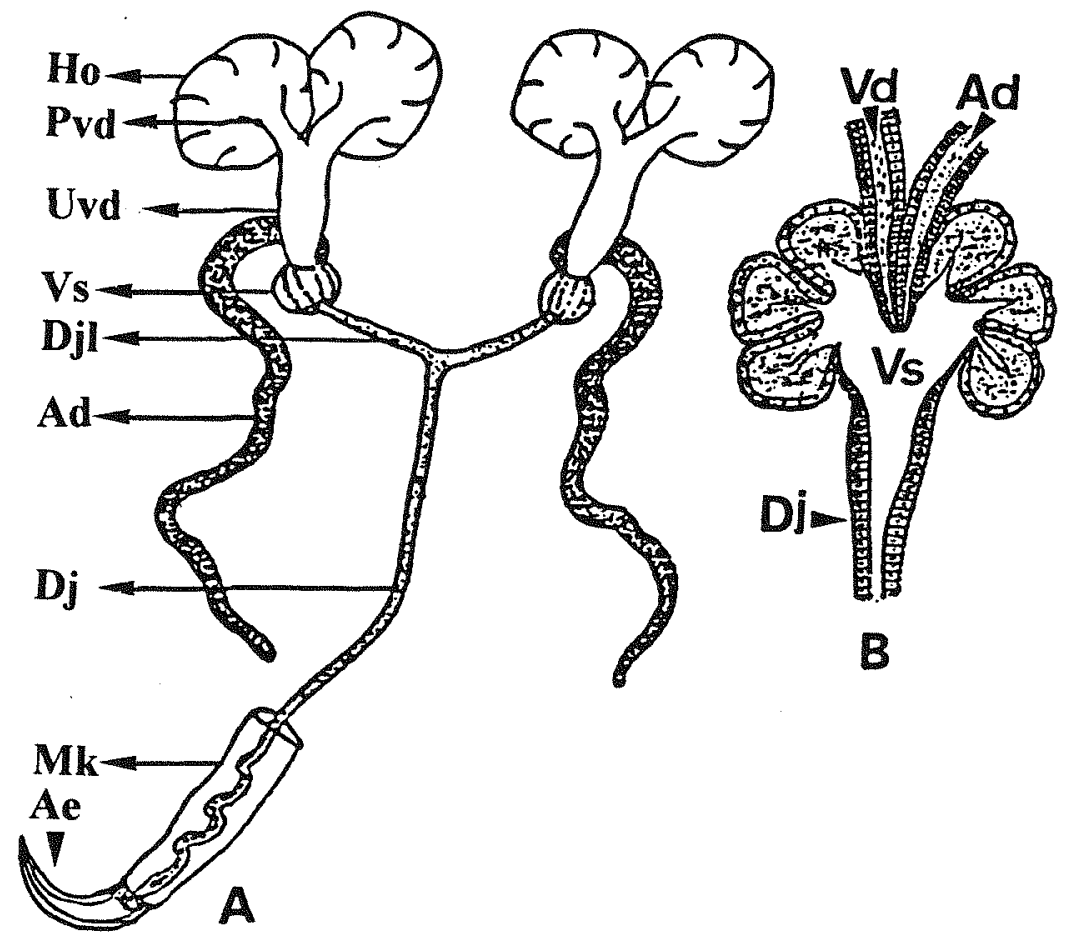

Fig. 1: Cosmopolites sordidus Schematische Darstellung des männlichen Geschlechtsapparates. A. Total, B. Ausschnitt. $\mathrm{Ad}=$ =akzessorische Drüse; $\mathrm{Ae}=$ =Aedeagus; $\mathrm{Dj}=$ Ductus ejaculatorius; $\mathrm{Djl}=$ Ductus ejaculatorius laterales; $\mathrm{Ho}=$ Hoden; $\mathrm{Mk}=$ Muskulatur des Kopulationsapparates; $\mathrm{Pvd}=$ paariges Vas deferens; Uvd=unpaariges Vas deferens; Vs=Vesicula seminales. 
Die paarigen Hoden zeigen eine Rosettenform und bestehen aus jeweils 5-6 Follikeln. Aus jedem Rosettenpaar geht ein Vas deferens hervor. Beide Vasa deferentia vereinigen sich nach kurzen Verlauf zu einem unpaaren Vas deferens. Das unpaare Vas deferens und die akzessorische Drüse münden auf gleicher Höhe in die kürbisförmige Vesicula seminales ein (Fig. 1B, 3A-B), an deren Ende beginnt der Ductus ejaculatorius (Fig. 1A-B). An dieser Einmündungsstelle kommen die durch die Vasa deferentia aus den Hoden austretenden Spermien sowohl mit dem Sekret des Vas deferens als auch mit dem Sekret der akzessorischen Drüse und der Vesicula seminales in Kontakt.

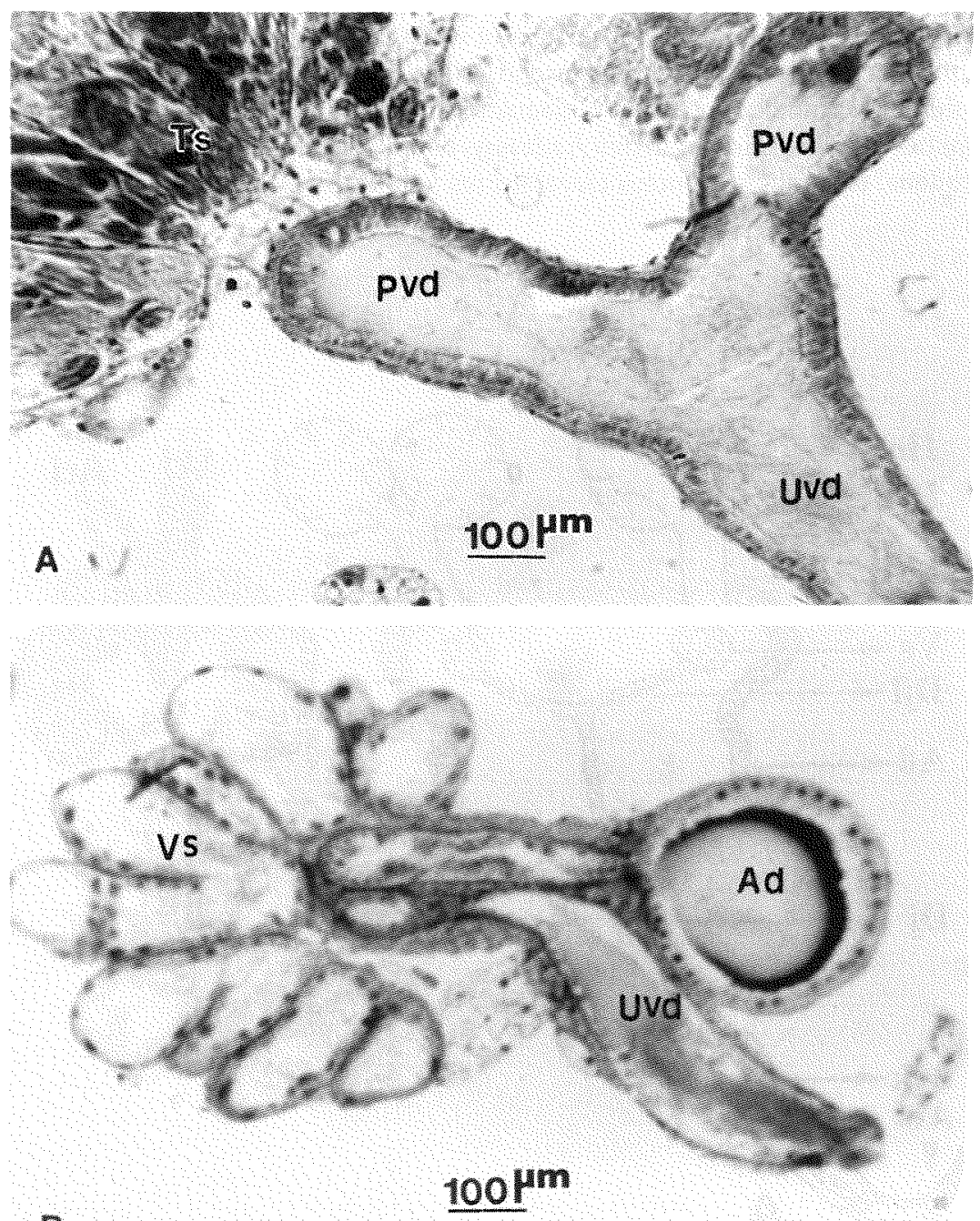

$\mathbf{B}$

Fig. 2: Cosmopolites sordidus Vasa deferentia, akzessorische Drüse. A-B. Längsschnitt durch den paarigen und den unpaaren Vas deferens mit akzessorischer Drüse. Ad: akzessorische Drüse, Pvd=paarige

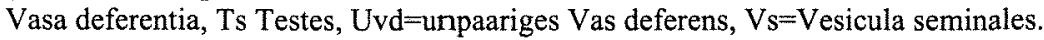


Das Epithel der Vasa deferentia besteht aus kubisch geformten Zellen mit großen runden, an der Basalseite der Zelle liegenden Kernen (Fig. 2A). Das Cytoplasma ist fein granuliert. Das Epithel ist von Muskulatur umhüllt. Das Lumen des Vas deferens ist mit Spermien und Sekret gefüllt (Fig. 2A-B). Das Sekret färbt sich mit Azan nach Heidenhain und Azan nach Specht (ROMEIS 1989) schwach blau. Das Sekret der Vasa deferentia und der akzessorische Drüsen zeigt mit BESTscher Karmin und PAS eine negative Reaktion auf Kohlenhydrate. Mit 1\%iger Osmiumsäure, Sudanschwarz B und Scharlach R zeigt sie keine Reaktion auf Lipide (SPANNHOF 1967). Das Sekret der Vasa deferentia und die akzessorische Drüse zeigen mit histochemischen Färbungen eine positive Reaktion auf Protein.

Die akzessorische Drüse von C. sordidus besteht aus zwei langen blind endenden Schläuchen, die durch ihr glänzendes Weiß auffallen (Fig. 4A). Die Drüsenzellen bilden eine hochprismatische Epithelschicht mit basal gelegenen großen runden Kernen (Fig. 5A-B). Die Epithelzellen sind von Ringmuskulatur umgeben. Die Zellgrenzen sind deutlich erkennbar. Das Cytoplasma ist fein granuliert. Das Lumen ist mit Sekret gefüllt. Das Sekret der akzessorischen Drüse, das sich mit Quecksilber-Bromphenol intensiv blau färbt, zeigt eine positive Reaktion auf Protein. Mit Hilfe der EIA-Daten errechnet sich die Proteinkonzentration der akzessorischen Drüse auf $1023 \mu \mathrm{g} / \mathrm{ml}$.

Die Beobachtung der blind endenden akzessorischen Drüsen von C. sordidus widerlegt demnach BECCARI (1967), der in der unpaaren akzessorische Drüse eine Verbindungsbrücke zwischen den beiden Ductus ejaculatorius gesehen hat. Auch hat BECCARI (1967) bei C. sordidus keine Vesicula seminalis nachgewiesen.

Fig. 3: Cosmopolites sordidus Vesicula seminales. A. Querschnitt, B. Ausschnitt; $\mathrm{Ad}=$ akzessorische Drüse, $\mathrm{Dj}=$ Ductus ejaculatorius, Uvd=unpaariges Vas deferens, $V_{s}=$ Vesicula seminales.
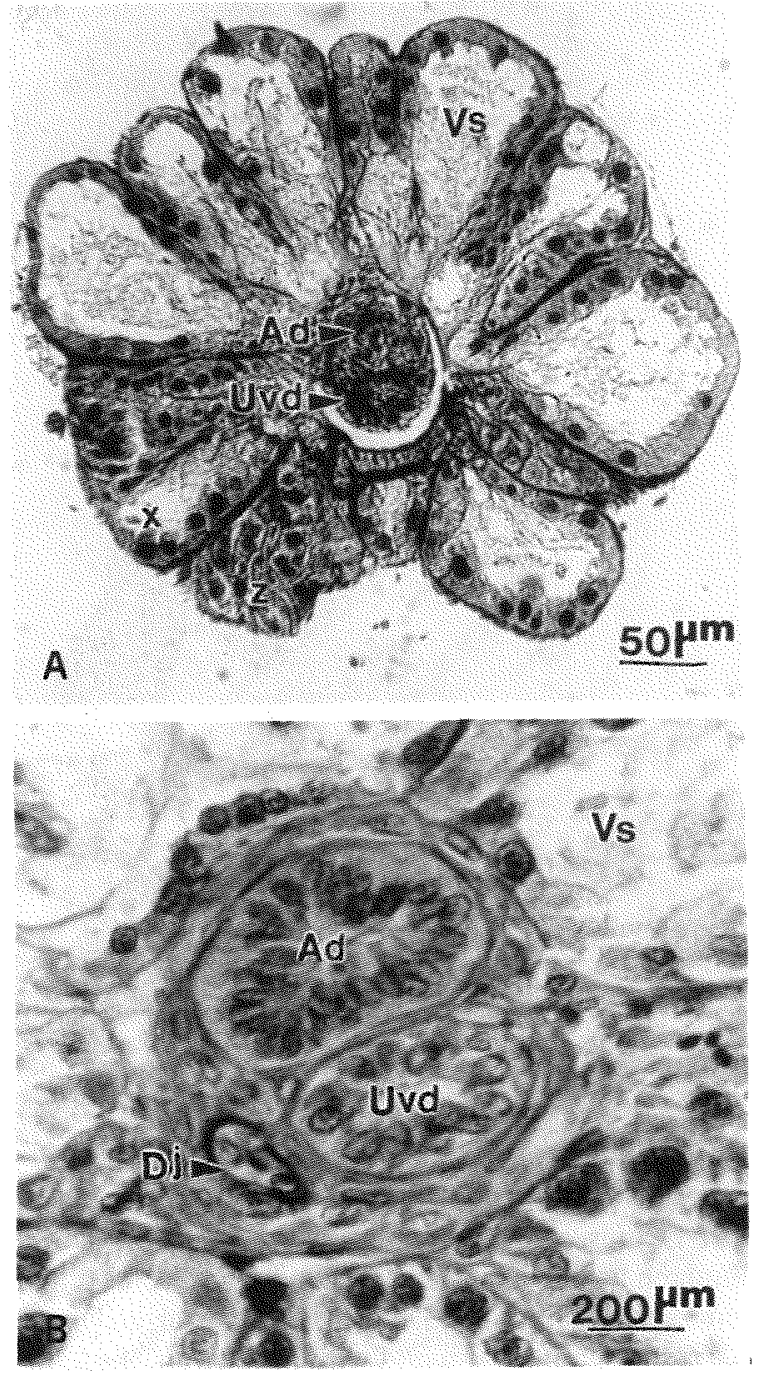

Die tortenförmigen Drüsenzellen der Vesicula seminales sind kubisch (Fig. 2B, Fig. 3A). Das kubische Epithel enthält große runde Kerne, die nahezu zentral im Cytoplasma liegen. Das Cytoplasma ist fein granuliert. Die Zellen produzieren ein feinkörniges Sekret, das über feine Kanälchen (Fig. 4B) abfließt. 


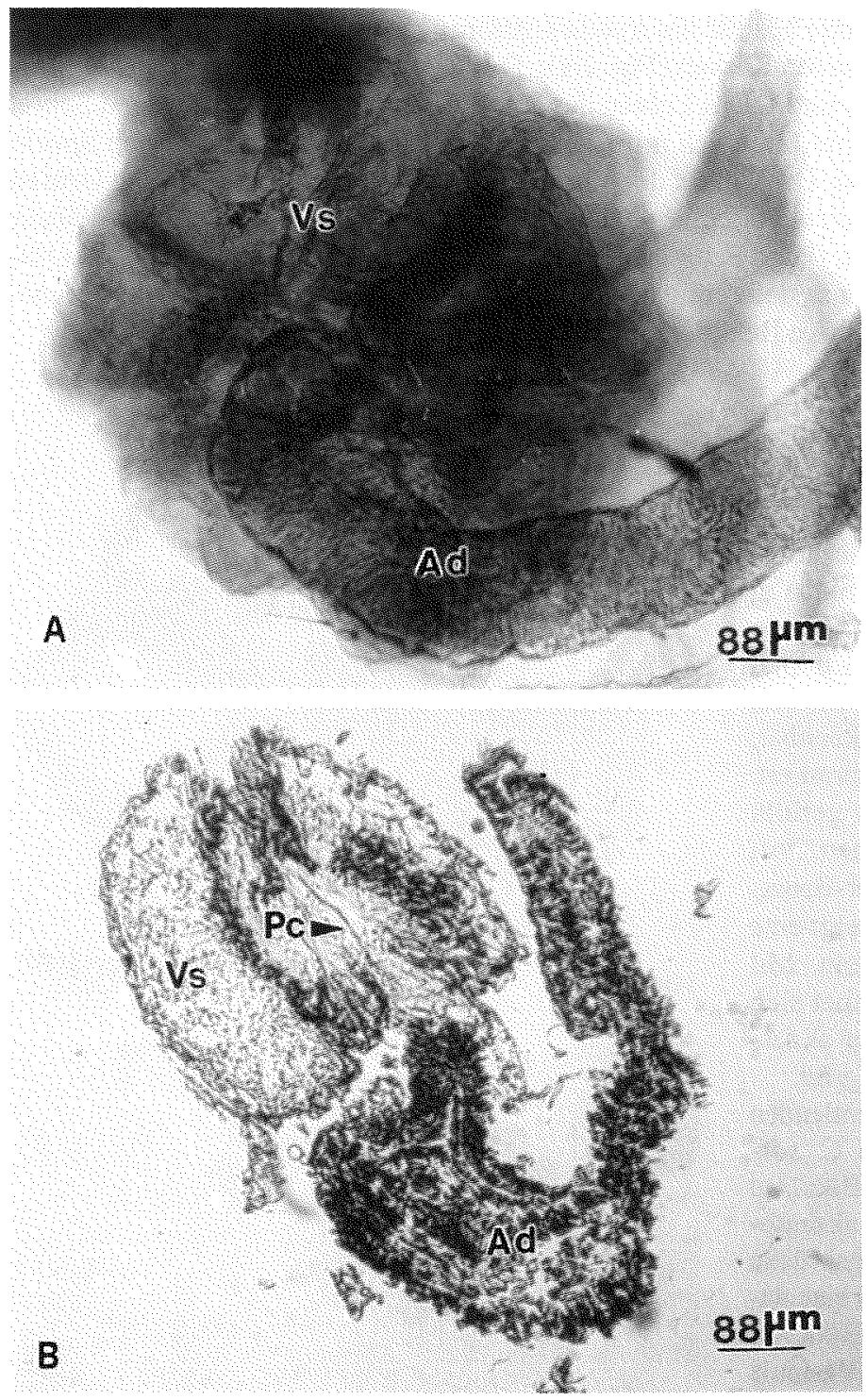

Fig. 4: Cosmopolites sordidus Akzessorische Drüse und Vesicula seminales. A. Total, B. Längsschnitt. $\mathrm{Ad}=$ akzessorische Drüse, $\mathrm{Pc}=$ Kanälchen, Vs $=$ Vesicula seminales.

Der Ductus ejaculatorius besteht aus zwei Teilen, dem vorderen paarigen kurzen und dem hinteren langen unpaarigen. Der paarige Ductus bildet als Übergang zum unpaarigen Abschnitt ein Dreieck (Fig. 1A). Der proximale, paarige Ductusabschnitt schließt sich beidseitig an die Vesicula seminales an und wird außen von Muskeln umkleidet. Im paarigen Ductusabschnitt besteht das Epithel aus kubischen Zellen mit großen runden Kernen. Die Kerne liegen nahezu zentral im Cytoplasma. Das Cytoplasma ist feinkörnig, die Zellgrenze ist deutlich erkennbar.Vom proximalen Teil des Ductus ejaculatorius (Fig. 6A) zu seinem kaudalen Ende hin (Fig. 6B) nehmen die Muskelschichten zu. 

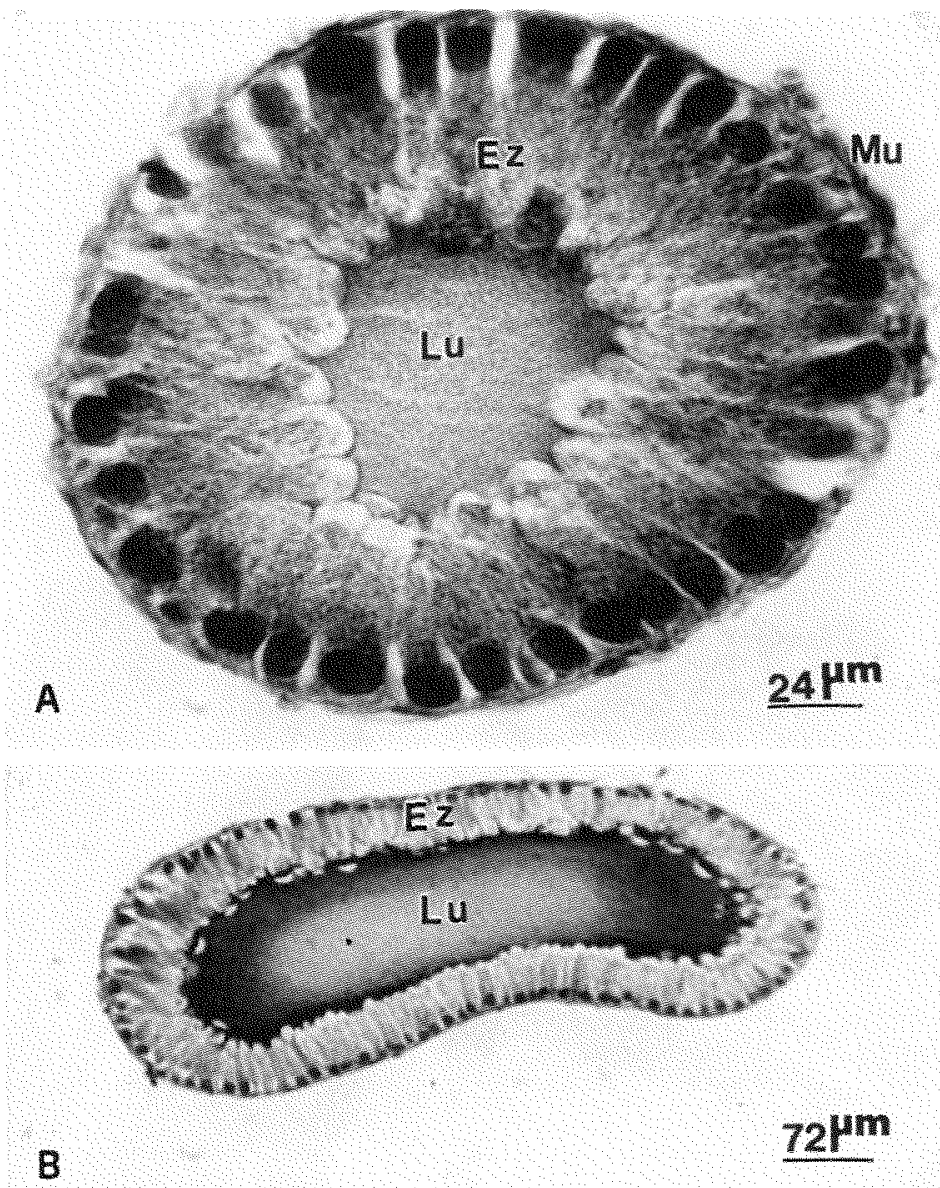

Fig. 5: Cosmopolites sordidus Akzessorische Drüse. A. Querschnitt, B. Längsschnitt. Ez=Epithelzelle, $\mathrm{Lu}=$ Lumen mit Sekret, $\mathrm{Mu}=$ Muskulatur.

Der hintere unpaarige Teil des Ductus ejaculatorius verläuft zum Hinterende in Windungen* (Fig. 6C), die von einer kräftigen 7-8 schichtigen Muskulatur umhüllt sind, während der vordere gegabelte Abschnitt nur von einer dünnen Muskelschicht umgeben ist (Fig. 6A). Der mit einer zart blau gefärbten kräftigen Kutikula (Fig. 6D) ausgestatte Ductus ejaculatorius ist $552 \mu \mathrm{m}$ lang. Sein terminaler Teil durchbohrt den kutikulären Aedeagus, das eigentliche Begattungsorgan. Im distalen Bereich ist das Epithel des Ductus ejaculatorius gefaltet, wodurch eine Dehnung des Kanals möglich ist.

Im Lumen konnten Spermien nachgewiesen werden. Dies bestätigt die DAPI-Färbung im histologischen Schnitt (Fig. 7B-C). Der sklerotisierte Aedeagus mißt ca. $62 \mu \mathrm{m}$ in der Länge. 

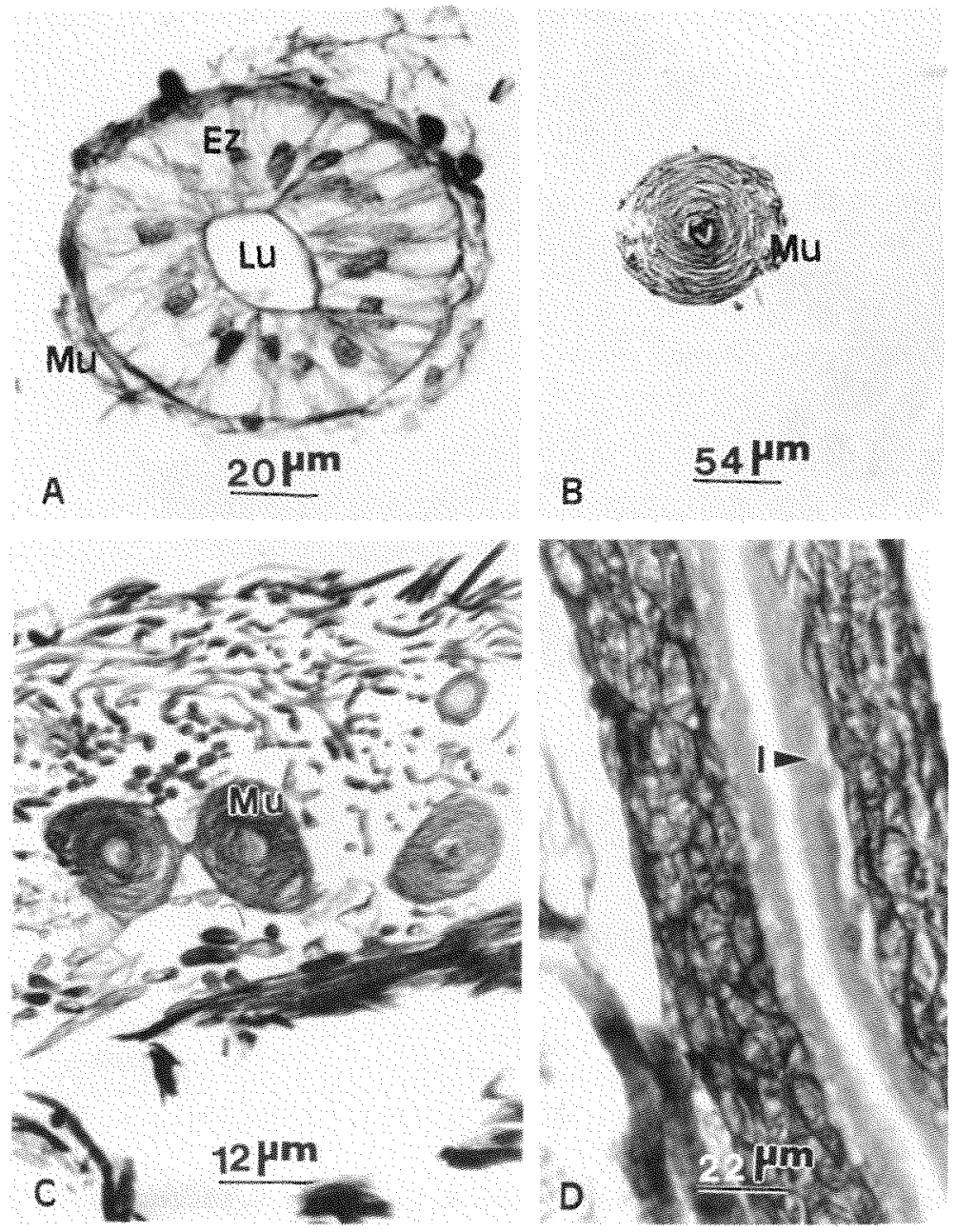

Fig. 6: Cosmopolites sordidus Querschnitt durch den Ductus ejaculatorius. A. vordere Abschnitt, B. Hinterer Abschnitt, C. Ausschnitt des gewundenen Ductus ejaculatorius (Querschnitt), D. Längsschnitt. Ez=Epithelzelle, I=Intima, Lu=Lumen, Mu=Muskulatur.

\section{Diskussion}

Die Geschlechtsapparat von $C$. sordidus wurde bisher kaum untersucht. Es liegt in der Literatur keine Angabe über die anatomische, histologische und histochemische Untersuchungen an $C$. sordidus vor. Detaillierte Untersuchungen über die Morphologie des Geschlechtsapparates von $C$. sordidus liegt von BECCARI (1967) und ZIMMERMANN (1968) vor.

Die Ergebnisse der Untersuchun gen am männlichen Geschlechtsapparat von C. sordidus zeigen, daß das Lumen des Vas deferens mit Spermium und Sekret gefüllt ist. Es wird vermutet, daß die Spermien bereits bei ihrer Wanderung aus den Hoden in den Vas deferens durch dessen Sekret aktiviert werden. 

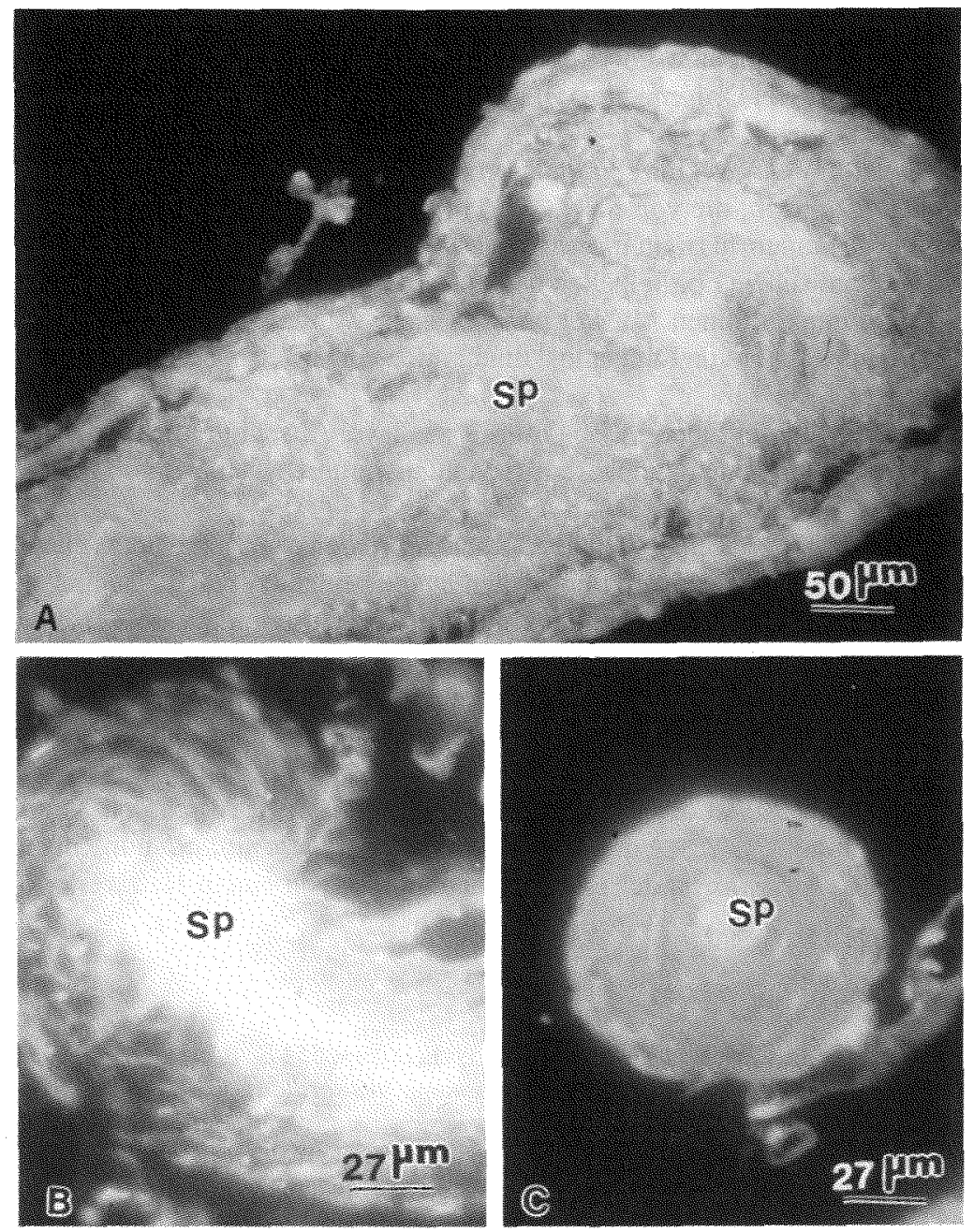

Fig. 7: Cosmopolites sordidus Vas deferens und Ductus ejaculatorius nach DAPI (Fluoreszensaufnahme). A. Vasa deferentia (Längsschnitt), B-C. Ductus ejaculatorious (Längs- und Querschnitt). Die Spermien $(=\mathrm{Sp})$ erscheinen weiß.

Das Sekret der Vasa deferentia und der akzessorische Drüsen zeigt eine deutliche negative Reaktion auf die Kohlenhydrate und Lipide. Mit Hilfe von histochemischen Färbungen konnten basische Proteine im Sekret der Vasa deferentia und der akzessorische Drüsen festgestellt werden. Die Proteinkonzenteration der akzessorische Drüse beläuft sich auf $1023 \mu \mathrm{g} / \mathrm{ml}$.

Nach BECCARI (1967) ist die akzessorische Drüse von $C$. sordidus unpaar und als Verbindungsbrücke zwischen beiden Ductus ejaculatorius des Hodens angelegt. Nach unseren Befunden kommen die akzessorischen Drüsen bei $C$. sordidus paarig vor und münden in die kürbisförmigen Vesicula seminales ein. 
Die Zahl der akzessorischen Drüsen ist bei den Insektenarten unterschiedlich. Bei Lytta muttalli sind drei Drüsenpaare entwickelt (GERBER u. a., 1971), bei Lilioceris lilii und Lilioceris merdigera zwei Drüsenpaare (BERTI \& RAPILLY 1976) und bei C. sordidus bestehen sie aus einem Drüsenpaar.

Aufbau und Struktur der Vesicula seminales von C. sordidus ist ähnlich der Drüse des Kornkäfers Calandra granaria, die von KRAUTWIG (1930) als Prostatadrüse bezeichnet wird und als Behälter für die Samenflüssigkeit dienen soll. Nach BECCARI (1967) fehlen bei C. sordidus die Vesicula seminales. Die Epithelzellen der Vesicula seminales sind bei C. sordidus, wie bei C. granaria, von einen dünnen Muskel umschlossen, während die Epithelzellen bei Parastizopus armaticepes außer von Längsmuskulatur noch von 2-3 Ringsmuskelschichten umhüllt sind (BRITS 1982).

Bei C. sordidus besteht der Ductus ejaculatorius aus zwei Abschnitten, einen vorderen paarigen, kurzen (laterale) und einem unpaaren, gewunden, hinteren Teil, der von kräftiger Muskulatur umgeben ist.

BERTI \& RAPILLY (1976) bezeichneten bei Lilioceris lilii den paarigen Ductus ejaculatorius als Ductus ejaculatorius lateral und den unpaarigen Ductus ejacuatorius als Ductus ejaculatorius commun. Außerdem bezeichneten sie die kürbisförmige Gestalt, die den Vesicula seminales von $C$. sordidus sehr ähnlich ist, als Vesicula seminales.

BECCARI (1967) bezeichnete bei $C$. sordidus den unpaaren Ductus ejaculatorius als primären Ductus ejaculatorius; das paarige und das unpaare Vas deferens als sekundären Ductus ejaculatorius.

Nach BEAURGARD (1890) ist der Ductus ejaculatorius von Lytta vesicatoria von Längs- und Ringmuskulatur umhüllt. GERBER u. a. (1971) konnten bei Lytta muttalli nur Ringsmuskulatur nachweisen. Bei $C$. sordidus ist der Ductus ejaculatorius von einem Netzwerk von Muskeln überzogen.

\section{Literatur}

BEAurgard, H. 1890: Les insectes Vesicants. - Paris: F. Alcan. 554 S.

BECCARI, F. 1967: Contributo alla conosenza del Cosmopolites sordidus (GERMAR) I. - Riv. Agric. Sub. Trop., Firenze 61: 51-93.

BENDICHO-LOPEZ, A. 1987: Poder de precepetion de las hormigas Tetamorium quinense para el control biologiaca del picudo negro del platano. - Cienc. Agric., Havana 30: 13-15.

BERTI, N. \& RAPILly, M. 1976: Faune d'Iran liste d'especes et revision du genre Lilioceris (REITTER) (Col. Chrysomelidae). - Ann. Soc. Ent. Fr., Paris (N.S.) 12 (1): 31-73.

BRITS, J. A. 1982: The anatomy, histology and physiology of the internal adult male reproductive system of Parastizopus armaticeps (Peringuey) (Col. Tenebrionidae). - J. Ent. Soc. South. Afr., Pretoria 45 (2): 239-260.

Castinerias, A.; Cabrera, T.; Calderon, A.; Lopez, M. \& Lujan, M. 1991: Lucha biologica contra Cosmopolites sordidus (GERMAR). - In: PAVIS, C. \& KEMARREC, A. (eds.), Rencontres carabies en lutte Biologique. - Proc. Symposium, 5-7 Nov. 1990, Gosier, Guadeloupe, Les Colleques de l'IRNA, Paris 58: 423-428.

CHAVARRIA-CARVAJAL, J. A. \& IRIZARRY, H. 1997: Rates, application intervals and rotation of four granular pesticides to control nematodes and the banana-weevil (Cosmopolites sordidus GERMAR) in plantain. - J. Agric. Unvi. Puerto-Rico, Rio Piedras 81 (1/2): 43-52.

FIGUEROA, W. 1990: Biocontrol of the banana root borer weevil, Cosmopolites sordidus (GERMAR) with steinernematid nematoides. - J. Agric. Univ. Puerto-Rico, Rio Piedras 74 (1): 15-19.

GERBER, G.; CHURCH, N. \& REMPEL, J. G. 1971: The anatomy, histology and physiology of the reproductive system of Lytta muttalli (SAY) (Col. Meloidae) I. The internal genitalia. - Can. J. Zool., Ottawa 49: 523-553. 
KOPPENHÖFFER, A. M. 1993: Observations on egg-laying behaviour of the banana weevil, Cosmopolites sordidus (GERMAR). - Ent. Exp. Appl., Amstardam 68: 187-192.

KRAUTWIG, M. 1930: Untersuchungen am Kornkäfer (Calandra granaria L.). Der Bau der Geschlechtsorgane und gegenseitige Korrelationen. - Zool. Jahrb. Abt. Anat., Jena 1: 539-596.

LIN, M. S.; CMINGS, D. E. \& ALFI, D. S. 1977: Optical studies of the interaction of 4-6-Diamidino-2phenylindole with DNA and metaphasa chromosomes. - Chromosoma, Berlin 60: 15-25.

LONGORIA, A. 1968: Diferencias sexuales en la morfologia externa de Cosmopolites sordidus GERMAR (Col.: Curcul.). - Cienc. Biol., La Habana 1: 1-11.

MAU, R. F. L. 1982; Banana root borer Cosmopolites sordidus (GERMAR) (Col.: Curcul.). - Horticulture digst. Univ. Hawaii Coop. Ext. Ser., Honolulu 66: 3-5.

MESQUITA, A. L. M. \& CALDAS, R. C. 1986: Efeito da idade de banaeira sobre a biologia e preferencia do Cosmopolites sordidus (GERMAR). - Fruits, Paris 41: 245-249.

NAHIF, A. A.; KOPPENHÖFFER, A. \& MADEL, G. 1994: Morphologie, Biologie und Bedeutung von Cosmopolites sordidus (Germar, 1824) (Col.: Curcul.). - Z. Angew. Zool., Berlin 4: 435-447.

NAIR, K. K. P. 1979: Studies of the chemical control of banana weevil (Cosmopolites sordidus, India). Note. - Agr. Res. J. Kerala, Trivandrum 17 (2): 280-281.

NARDON, P.; KERMARREC, A. \& NARDON, C. 1984: Caractares morphologiques distinctif des larves de Cosmopolites sordidus (GERMAR) et Metamasius hemipterus (LINNE) (Col.: Curcul.), parasites du bananier. - Fruits, Paris 39 (3): 180-187.

ROMEIS, B. 1989: Mikroskopische Technik, 17. Aufl, München, Urban \& Schwarzenberg, 697 S.

RUTHMANN, A.1966: Methoden der Zellforschung, Stuttgart. - Franckhsche Verlagsbuchhandlung, $301 \mathrm{~S}$.

SCHOEMAN, S. 1998: Chemical control of the banana root borer. - Neltropika-Bulletin, Nelsprnit 301: 28-30.

SPANNHOF, L. 1967: Einführung in die Praxis der Histochemie, Jena. - G. Fischer Verl. $172 \mathrm{~S}$.

VISNAWATH, B. N. 1981: Development of Cosmopolites sordidus (Col.: Curcul.) on banana varieties in south India. - Colemania, Bangalore 1: 57-58.

ZIMMERMANN, E. C. 1968: The Cosmopolites banana weevils (Coleoptera: Curculionidae: Rhynchophorinae). - Pacific Insects, Honolulu 10 (2): 295-299.

\author{
Anschrift des Verfassers: \\ Prof. Dr. ALI AGHA NAHIF \\ Institut für Evolutionsbiologie und \\ Ökologie der Universität Bonn \\ An der Immenburg 1 \\ D-53121 Bonn \\ Deutschland
}

\title{
Besprechungen
}

Phylogeny and Evolution of Subterranean and Endogean Cholevidae (= Leiodidae, Cholevinae). Proceedings of a Symposium (30 August, 1996, Florence, Italy). XX International Congress of Entomology / Pier Mauro Giachino \& SteWART B. PEck Editors. - Atti Museo Regionale di Scienze Naturali, Torino 1998. - 295 S.: zahlr. Abb. - ISSN 1123-1246, ISBN 88-86041-32-2. - £110.000

Die subterranen und endogäischen Leiodidae, Cholevinae (oder Cholevidae) sind wegen ihrer Adaptationen an die unterirdische Lebensweise nicht nur innerhalb der Staphylinoidea eine faszinierende Gruppe, der sich auch der Rezensent in der Vergangenheit nicht ganz entziehen konnte. Als Beleg dafür können auch die einzelnen Beiträge des vorliegenden Sammelbandes mit ihren Abbildungen dienen.

STEWART B. PECK gibt eine Einführung in die Phylogenie und Evolution der subterranen und endogäischen Cholevidae (S. 11-40). Im Zusammenhang mit einer weltweiten Bearbeitung der Klassifikation der Staphylinoidea werden von ALFRED F. NEWTON die gegenwärtige Klassifikation der Leiodidae oberhalb 